\title{
On Computing J-Inner-Outer Factorizations of Rational Matrices
}

\author{
A. Varga \\ German Aerospace Research Establishment \\ Institute of Robotics and System Dynamics \\ D-82234 Wessling, Germany \\ Fax: +49-8153-28-1441 \\ Andreas.Varga@dlr.de
}

\author{
T. Katayama \\ Dept. Applied Mathematics and Physics \\ Faculty of Engineering, Kyoto University \\ Kyoto, 606-01, Japan \\ Fax: +81-75-761-2437 \\ katayama@kuamp.kyoto-u.ac.jp
}

\begin{abstract}
In this paper we propose a new numerically reliable computational approach to determine the J-innerouter factorization of a rational matrix $G$. The proposed approach is completely general being applicable whenever $G$ is proper or not, or of full column/row rank or not. In contrast to existing "one-shot" methods which require the solution of Riccati or generalized Riccati equations, the new approach is recursive and avoids such computationally involved steps by using instead a recursive state-space approach. The resulting factors have always minimal order descriptor representations.
\end{abstract}

\section{Introduction}

Let $G(\lambda)$ be a given $p \times m$ stable rational transferfunction matrix (TFM) of a linear time-invariant continuous-time or discrete-time descriptor system and let $G=(E, A, B, C, D)$ denote an equivalent $n$th order regular $(\operatorname{det}(\lambda E-A) \not \equiv 0)$ irreducible descriptor representation satisfying $G(\lambda)=$ $C(\lambda E-A)^{-1} B+D$, where $\lambda$ is either $s$ or $z$, depending on the type of the system. We assume that $G$ has no zeros on the imaginary axis for a continuous-time system or on the unit circle circle for a discrete-time system. In this paper we address the problem to compute a $J$-inner-outer factorization of $G$, namely $G=G_{i} G_{o}$, where $G_{i}$ is a square J-inner factor and $G_{o}$ is an outer factor. Recall that for a given inertia matrix $J, G_{i}$ is $J$-inner means $G_{i}$ is stable and $G_{i}^{*} J G_{i}=J$, where $G_{i}^{*}(s)=G_{i}^{T}(-s)$ in continuous-time and $G_{i}^{*}(z)=G_{i}^{T}(1 / z)$ in discrete-time. In this paper, $G_{o}$ is outer means that it has only stable poles and zeros. This definition of outer matrices extends, by a slight abuse of language, the standard definition [1] (applicable to full row rank matrices), to rational matrices of arbitrary rank.

In this paper we propose a new numerically reliable computational approach to compute the J-innerouter factorization of $G$. In contrast to several "one-shot" methods [2], [3], [4], which require the solution of Riccati or generalized Riccati equations, our approach is recursive and avoids such computationally involved steps by using instead a recursive state-space approach. Generalized Lyapunov equations of order at most two are solved repeatedly to compute suitable elementary J-inner factors to reflect the unstable zeros into the stable region of the complex plane. The proposed approach is completely general being applicable whenever $G$ is proper or not, or of full column/row rank or not. The resulting J-inner and outer factors have always minimal order descriptor representations.

The procedure to compute J-inner-outer factorizations is conceptually similar to that of [5] to compute inner-outer factorizations and has the following main steps:

1. Compute a generalized inverse $G^{+}$of $G$ such that the unstable poles of $G^{+}$are exactly the unstable zeros of $G$.

2. Compute a right coprime factorization with $J$ inner denominator (RCFJID) of $G^{+}$as $G^{+}=$ $N G_{i}^{-1}$, where $N$ and $G_{i}$ are stable TFMs with $G_{i}$ J-inner and of least order.

3. Compute $G_{o}=G_{i}^{-1} G$.

If the generalized inverse $G^{+}$computed at step 1 is an (1,2)-generalized inverse satisfying $G G^{+} G=G$ 
and $G^{+} G G^{+}=G^{+}[6]$, then it is easy to see that the computed $G_{o}$ is outer. This follows by observing that $N$ is a stable $(1,2)$-generalized inverse of $G_{o}$, satisfying $G_{o} N G_{o}=G_{o}$ and $N G_{o} N=N$. The requirements at step 1 that $G^{+}$has the only unstable poles, the unstable zeros of $G$, and at step 2 that $G_{i}$ has least order ensure that the order of the resulting J-inner factor $G_{i}$ equals the number of unstable zeros of $G$. The requirement for the least order of $G_{i}$ is a necessary condition to compute the corresponding $G_{o}$ because the need for unstable poles-zeros cancellation in computing $G_{i}^{-1} G$ at step 3. The existence of the J-inner-outer factorization is implicitly verified by the procedure to compute $G_{i}$.

In the rest of the paper we discuss in detail the main computational problems appearing in the proposed computational approach.

\section{Computation of generalized inverses}

In this section we discuss a numerically reliable approach to compute a descriptor representation of a particular $(1,2)$-generalized inverse $G^{+}$fulfilling the requirement to have as unstable poles the unstable zeros of $G$. It is known that for a non-square full rank TFM $G$ the zeros of $G$ (finite and infinite) are always poles of any left or right inverse $G^{+}$ [7, page 467]. However besides these fixed poles $G^{+}$generally possesses also spurious poles resulting from the particular way the inverse was determined. In order to simplify the procedure at step 2 , it is convenient to devise an inversion procedure by which these spurious poles result always stable. This allows an easy computation of a least order inner denominator (if exists) at step 2.

Consider system matrix pencil

$$
\mathcal{S}(\lambda)=\left[\begin{array}{c|c}
A-\lambda E & B \\
\hline C & D
\end{array}\right] .
$$

associated to the descriptor representation $G=$ $(E, A, B, C, D)$. The computation of an $(1,2)-$ generalized inverse of $G$ relies on the following straightforward formula [8]

$$
G^{+}(\lambda)=\left[\begin{array}{ll}
0 & I_{m}
\end{array}\right] \mathcal{S}(\lambda)^{+}\left[\begin{array}{c}
0 \\
I_{p}
\end{array}\right]
$$

where $\mathcal{S}(\lambda)^{+}$is an $(1,2)$-generalized inverse of $\mathcal{S}(\lambda)$. It can be readily verified that if $\mathcal{S}(\lambda)^{+}$is an $(1,2)$ generalized inverse then the corresponding $G^{+}(\lambda)$ in (2) is also an (1,2)-generalized inverse [8].
With the help of two orthogonal matrices $Q$ and $Z$ it is possible to reduce the system pencil (1) to the following Kronecker-like form which exhibits the complete Kronecker structure of $\mathcal{S}(\lambda)$ :

$$
\begin{gathered}
\mathcal{S}_{1}(\lambda):=Q \mathcal{S}(\lambda) Z= \\
{\left[\begin{array}{c|ccc}
B_{r} & A_{r}-\lambda E_{r} & * & * \\
0 & 0 & A_{r e g}-\lambda E_{r e g} & * \\
0 & 0 & 0 & A_{l}-\lambda E_{l} \\
\hline 0 & 0 & 0 & C_{l}
\end{array}\right],}
\end{gathered}
$$

where: 1) the pencil [ $B_{r} A_{r}-\lambda E_{r}$ ] with $E_{r}$ nonsingular contains the right Kronecker structure of $\mathcal{S}(\lambda)$ and the pair $\left(B_{r}, A_{r}-\lambda E_{r}\right)$ is controllable; 2) the regular pencil $A_{r e g}-\lambda E_{r e g}$ has the form

$$
A_{r e g}-\lambda E_{r e g}=\left[\begin{array}{cc}
A_{\infty}-\lambda E_{\infty} & * \\
0 & A_{f}-\lambda E_{f}
\end{array}\right]
$$

where the pencil $A_{\infty}-\lambda E_{\infty}$ with $A_{\infty}$ non-singular and $E_{\infty}$ nilpotent contains the infinity Kronecker structure of $\mathcal{S}(\lambda)$, while the pencil $A_{f}-\lambda E_{f}$ with $E_{f}$ non-singular contains the finite Kronecker structure of $\mathcal{S}(\lambda)$; the generalized eigenvalues of the pair $\left(A_{f}, E_{f}\right)$ are the finite zeros of the $\left.G ; 4\right)$ the pencil $\left[\begin{array}{c}A_{l}-\lambda E_{l} \\ C_{l}\end{array}\right]$ with $E_{l}$ non-singular contains the left Kronecker structure of $\mathcal{S}(\lambda)$ and the pair $\left(C_{l}, A_{l}-\lambda E_{l}\right)$ is observable.

By using additional left and right transformation matrices $U$ and $V$ of the special forms

$$
U=\left[\begin{array}{cccc}
I & 0 & 0 & 0 \\
0 & I & 0 & 0 \\
0 & 0 & I & K \\
0 & 0 & 0 & I
\end{array}\right], \quad V=\left[\begin{array}{llll}
I & F & 0 & 0 \\
0 & I & 0 & 0 \\
0 & 0 & I & 0 \\
0 & 0 & 0 & I
\end{array}\right]
$$

we obtain

$$
\begin{gathered}
\widetilde{\mathcal{S}}(\lambda)=\left[\begin{array}{c|cc}
\widetilde{\mathcal{S}}_{11}(\lambda) & \widetilde{\mathcal{S}}_{12}(\lambda) \\
\hline 0 & \widetilde{\mathcal{S}}_{22}(\lambda)
\end{array}\right]:=U \mathcal{S}_{1}(\lambda) V= \\
{\left[\begin{array}{c|ccc}
B_{r} & A_{r}+B_{r} F-\lambda E_{r} & * & * \\
0 & 0 & A_{r e g}-\lambda E_{r e g} & * \\
0 & 0 & 0 & A_{l}+K C_{l}-\lambda E_{l} \\
\hline 0 & 0 & 0 & C_{l}
\end{array}\right]}
\end{gathered}
$$

Because the pair $\left(B_{r}, A_{r}-\lambda E_{r}\right)$ is controllable and the pair $\left(C_{l}, A_{l}-\lambda E_{l}\right)$ is observable, we can arbitrarily assign stable spectrums for the matrix pairs $\left(A_{r}+B_{r} F, E_{r}\right)$ and $\left(A_{l}+K C_{l}, E_{l}\right)$ by choosing suitable state-feedback and output-injection matrices $F$ and $K$, respectively.

With the partitioning of $\widetilde{\mathcal{S}}(\lambda)$ as in (6) it follows that for almost all $\lambda, \operatorname{rank} \mathcal{S}(\lambda)=\operatorname{rank} \widetilde{\mathcal{S}}_{12}(\lambda)$, and 
thus a generalized $(1,2)$-inverse of $\mathcal{S}(\lambda)$ can be computed as [6]

$$
\mathcal{S}(\lambda)^{+}=Z V\left[\begin{array}{cc}
0 & 0 \\
\widetilde{\mathcal{S}}_{12}(\lambda)^{-1} & 0
\end{array}\right] U Q .
$$

To compute a descriptor representation of the generalized inverse $G(\lambda)^{+}$it is not necessary to explicitly evaluate $\widetilde{\mathcal{S}}_{12}(\lambda)^{-1}$. If we denote

$$
\begin{gathered}
\widetilde{A}_{12}-\lambda \widetilde{E}_{12}=\widetilde{\mathcal{S}}_{12}(\lambda), \quad \widetilde{B}=U Q\left[\begin{array}{c}
0 \\
I_{p}
\end{array}\right]=\left[\begin{array}{c}
\widetilde{B}_{1} \\
\widetilde{B}_{2}
\end{array}\right] \\
\widetilde{C}=\left[\begin{array}{ll}
0 & I_{m}
\end{array}\right] Z V=\left[\widetilde{C}_{1} \widetilde{C}_{2}\right],
\end{gathered}
$$

where $\widetilde{B}$ and $\widetilde{C}$ are partitioned analogously with the column and row partition of $\widetilde{\mathcal{S}}(\lambda)$ in $(6)$, respectively, then the corresponding $G(\lambda)^{+}$is given by

$$
G(\lambda)^{+}=-\widetilde{C}_{2}\left(\lambda \widetilde{E}_{12}-\widetilde{A}_{12}\right)^{-1} \widetilde{B}_{1}
$$

and thus $\left(\widetilde{E}_{12}, \widetilde{A}_{12}, \widetilde{B}_{1},-\widetilde{C}_{2}, 0\right)$ is a descriptor representation of $G(\lambda)^{+}$.

From the above construction it is clear that the poles of $G^{+}$include always the zeros of $G$ (the generalized eigenvalues of the regular part (4)) as the fixed poles of $G^{+}$. The spurious poles are the union of generalized eigenvalues of the pairs $\left(A_{r}+B_{r} F, E_{r}\right)$ and $\left(A_{l}+K C_{l}, E_{l}\right)$ and as mentioned before, can be arbitrarily assigned. If $G$ has only stable zeros, then this approach can be used to compute stable generalized inverses of $G$ by appropriately determining stabilizing matrices $F$ and $K$. Generally with $F$ and $K$ stabilizing, the only unstable poles of the generalized inverse (8) are the unstable zeros of $G$. Thus $G(\lambda)^{+}$satisfies the condition imposed at step 1 of the procedure proposed in the previous section having the only unstable poles the unstable zeros of $G$.

To compute at step 3 the outer factor $G_{o}$ only the J-inner denominator $G_{i}$ in the RCFJID $G^{+}=$ $N G_{i}^{-1}$ is necessary. As it will be apparent in the next section, in this case the output matrix $-\widetilde{C}_{2}$ of $G^{+}$plays no role in the computations. In fact, to compute $G_{i}$ it is sufficient to have $\widehat{A}-\lambda \widehat{E}$, the lower right corner of $\widetilde{A}_{12}-\lambda \widetilde{E}_{12}$ defined as

$$
\widehat{A}-\lambda \widehat{E}=\left[\begin{array}{cc}
A_{f}-\lambda E_{f} & * \\
0 & A_{l}+K C_{l}-\lambda E_{l}
\end{array}\right],
$$

and $\widehat{B}$, the corresponding rows of the input matrix $\widetilde{B}_{1}$. Thus the computation of the right transformation matrices $Z$ and $V$ is not necessary. Moreover, instead of the more complex Kronecker-like form
(3), the following simpler Kronecker-like form can be used

$$
\begin{gathered}
\mathcal{S}_{2}(\lambda):=Q \mathcal{S}(\lambda) Z= \\
{\left[\begin{array}{ccc}
A_{r}-\lambda E_{r} & * & * \\
0 & A_{f}-\lambda E_{f} & * \\
0 & 0 & A_{l}-\lambda E_{l} \\
\hline 0 & 0 & C_{l}
\end{array}\right],}
\end{gathered}
$$

where this time the pencil $A_{r}-\lambda E_{r}$ contains the right and the infinity Kronecker structures of the system pencil.

The reduction techniques to compute (3) or (10) is based on structure preserving algorithms similar to those described in [9] to compute the system zeros. A complete algorithm to compute (3) is described in detail in [10] together with the corresponding computational programs. The simpler form (10) is an intermediary step in computing (3). The stabilizing matrix $K$ in (9) can be efficiently computed by using either direct stabilization methods or pole assignment techniques for descriptor systems as those proposed in [11]. The computational complexity of computing a generalized inverse of $G$ is $0\left(n^{3}\right)$ and all computations can be done with numerically stable or numerically reliable algorithms.

\section{Computation of the J-inner factor}

Let $G=(E, A, B, C, D)$ be a stabilizable descriptor representation of a rational TFM $G$ and let $J=$ $\operatorname{diag}\left(I_{m_{1}},-I_{m_{2}}\right)$ be a given inertia matrix such that $m_{1}+m_{2}=m$, where $m$ is the number of columns of $G$. In this section we present an algorithm to compute a least order J-inner denominator $G_{i}$ of a RCFJID of $G, G=N G_{i}^{-1}$. This algorithm can be used at step 2 of the proposed approach to determine the least order J-inner denominator factor of the RCFJID of $G^{+}$. The algorithm to compute $G_{i}$ is similar to that proposed in [12] to compute right coprime factorizations with inner denominators and relies on several simple facts.

Fact 1. [13] For any $F$ of appropriate dimensions and for any invertible $W$, the descriptor representations

$$
\begin{aligned}
& N=(E, A+B F, B W, C+D F, D W) \\
& M=(E, A+B F, B W, F, W)
\end{aligned}
$$

give a fractional representation of $G$ as $G=$ $N M^{-1}$. If $F$ is chosen such that the pair $(E, A+$ $B F)$ is stable, then the fractional representation is a right coprime factorization.

It is apparent that in computing the denominator factor $M$ the output matrix $C$ plays no role. This 
fact leads to a sensible simplification of the procedure presented in section 1 .

The algorithm to compute J-inner denominators uses recursively the following explicit updating technique of fractional representations.

Fact 2. If $G=N_{1} M_{1}^{-1}$ and $N_{1}=N_{2} M_{2}^{-1}$ are fractional representations with $J$-inner denominators, then $G$ has the fractional representation $G=N M^{-1}$, where $N=N_{2}$ and $M=M_{1} M_{2}$ is J-inner.

This simple fact allows us to obtain explicit formulas to update partial factorizations by using simple state space formulas. Let $N_{1}$ and $M_{1}$ be the factors computed as

$$
\begin{aligned}
& N_{1}=\left(E, A+B F_{1}, B W_{1}, C+D F_{1}, D W_{1}\right) \\
& M_{1}=\left(E, A+B F_{1}, B W_{1}, F_{1}, W_{1}\right)
\end{aligned}
$$

and let $N_{2}$ and $M_{2}$ be the factors of $N_{1}$ computed as

$$
\begin{aligned}
& N_{2}=(E, A+B F, B W, C+D F, D W) \\
& M_{2}=\left(E, A+B F, B W, F_{2}, W_{2}\right)
\end{aligned}
$$

where

$$
\begin{aligned}
& F=F_{1}+W_{1} F_{2} \\
& W=W_{1} W_{2}
\end{aligned}
$$

It easy to verify that the product $M_{1} M_{2}$ is given by

$$
M_{1} M_{2}=(E, A+B F, B W, F, W)
$$

and thus the equations (11) and (12) serve as explicit updating formulas of fractional representations.

Elementary first or second order J-inner factors can be used to reflect the unstable poles of $G$ to symmetric positions with respect to the imaginary axis in case of continuous-time systems or with respect of the unit circle in case of discrete-time systems. This pole dislocation technique is used in our algorithm and is conceptually similar to the conjugation technique proposed in [2]. Formulas to compute elementary J-inner denominators are established below.

Fact. 3 Let $G=(E, A, B, *, *)$ a controllable descriptor representation with $E$ non-singular and $\Lambda(E, A) \in \mathbb{C}^{+}$(the appropriate unstable region of the complex plane $\mathbb{C})$. Then the denominator factor $G_{i}=(E, A+B F, B W, F, W)$ is J-inner by choosing $F$ and $W$ as:

$$
\begin{aligned}
& A Y E^{T}+E Y A^{T}-B J B^{T}=0 \\
& F=-J B^{T}\left(Y E^{T}\right)^{-1}, \quad W=I,
\end{aligned}
$$

for a continuous-time system, and

$$
\begin{aligned}
& A Y A^{T}-B J B^{T}=E Y E^{T} \\
& F=-J B^{T}\left(E Y E^{T}+B J B^{T}\right)^{-1} A \\
& W^{T}\left(J+B^{T}\left(E Y E^{T}\right)^{-1} B\right) W=J,
\end{aligned}
$$

for a discrete-time system, where $Y$ must be invertible.

The invertibility of $Y$ guarantees the existence of a least order J-inner denominator. (Notice that a RCFJID of non-least order always exists.) If supplementary $Y>0$, then the J-inner factor is also lossless [4]. In computing the elementary J-inner factors the invertibility (or positive definiteness) of $Y$ must be checked. If this check fails, then the given TFM has no RCFJID with the J-inner denominator of least order.

In the discrete-time case, $W$ can be computed easily by observing that the symmetric matrices $Z=J+B^{T}\left(E Y E^{T}\right)^{-1} B$ and $J$ must have the same inertia. Thus if $Z=U G U^{T}$ is the orthogonal symmetric decomposition of $Z$ with $G$ diagonal and $G J>0$, then $W$ is simply $W=U(G J)^{-1 / 2}$.

\section{RCFJID Algorithm.}

1. Find orthogonal matrices $Q$ and $Z$ to reduce the pair $(E, A)$ to the ordered generalized real Schur form (GRSF) (QAZ, $Q E Z)$ such that

$Q E Z=\left[\begin{array}{cc}E_{11} & E_{12} \\ 0 & E_{22}\end{array}\right], Q A Z=\left[\begin{array}{cc}A_{11} & A_{12} \\ 0 & A_{22}\end{array}\right], Q B=\left[\begin{array}{l}B_{1} \\ B_{2}\end{array}\right]$

where $E_{22}, A_{22} \in \mathbb{R}^{q \times q}, \Lambda\left(A_{11}, E_{11}\right) \cap \mathbb{C}^{+}=\phi$ and $\Lambda\left(A_{22}, E_{22}\right) \subset \mathbb{C}^{+}$.

Set $(\widehat{A}, \widehat{E}, \widehat{B}, \widehat{F}, \widehat{W})=\left(A_{22}, E_{22}, B_{2}, 0, I\right), i=1$.

2. If $q=0$, go to 6 .

3 . Let $(\delta, \alpha)$ be the last diagonal blocks of $(\widehat{E}, \widehat{A})$ of order $k$ and let $\beta$ be the $k \times m$ matrix formed from the last $k$ rows of $\widehat{B}$. For the system $(\delta, \alpha, \beta, *, *)$ compute $\varphi, V$ and $Y$ (the solution of the corresponding Lyapunov equation) such that $(\delta, \alpha+\beta \varphi, \beta V, \varphi, V)$ is J-inner. If $Y$ is not invertible (or not positive definite), then error and exit; else, set $K=[0 \varphi]$.

4. Compute $\widehat{A} \leftarrow \widehat{A}+\widehat{B} K, \widehat{F} \leftarrow \widehat{F}+\widehat{W} K$, $\widehat{W} \leftarrow \widehat{W} V$.

5. Compute the orthogonal $\widehat{Q}$ and $\widehat{Z}$ to move the last blocks of $(\widehat{E}, \widehat{A})$ to positions $(i+1, i+1)$ by interchanging the diagonal blocks of the GRSF. Compute $\widehat{E} \leftarrow \widehat{Q} \widehat{E} \widehat{Z}, \widehat{A} \leftarrow \widehat{Q} \widehat{A} \widehat{Z}, \widehat{B} \leftarrow \widehat{Q} \widehat{B}$, $\widehat{F} \leftarrow \widehat{F} \widehat{Z}$. Put $q \leftarrow q-k, i \leftarrow i+k$ and go to 2 .

6. Set $G_{i}=(\widehat{E}, \widehat{A}, \widehat{B} \widehat{W}, \widehat{F}, \widehat{W})$. 
If the RCFJID Algorithm is applied to the TFM $G^{+}$computed in the previous section, then the order of the J-inner factor is precisely the number of unstable zeros of $G$. The resulting pair $(\widehat{E}, \widehat{A})$ is in a generalized real Schur form having as stable eigenvalues the reflected unstable zeros of $G$.

The RCFJID algorithm relies on reliable numerical techniques. It can be viewed as a pole assignment algorithm (similar to that of [11]) which assigns the unstable poles in symmetrical positions with respect to the imaginary axis in the continuoustime case or the unit circle in the discrete-time case. Because practically there is no freedom in assigning the poles, it is to be expected that the algorithm perform in a numerically stable way only if the norms of the elementary feedback matrices $K$ computed at step 3 are not too high.

\section{Computation of outer factor}

The outer factor $G_{o}$ can be computed by removing the uncontrollable unstable eigenvalues from the descriptor representation of $G_{i}^{-1} G$ :

$$
\left(\left[\begin{array}{cc}
\widehat{E} & 0 \\
0 & E
\end{array}\right],\left[\begin{array}{cc}
\widehat{A}-\widehat{B} \widehat{F} \widehat{B} C \\
0 & A
\end{array}\right],\left[\begin{array}{c}
\widehat{B} D \\
B
\end{array}\right],\left[-\widehat{W}^{-1} \widehat{F} C\right], \widehat{W}^{-1} D\right)
$$

These eigenvalues can be removed in a numerically sound way by using the following approach. First reduce the pair

$$
\left(\left[\begin{array}{cc}
\widehat{A}-\widehat{B} \widehat{F} & \widehat{B} C \\
0 & A
\end{array}\right],\left[\begin{array}{cc}
\widehat{E} & 0 \\
0 & E
\end{array}\right]\right)
$$

by using an orthogonal similarity transformation, to an ordered GRSF where the unstable diagonal blocks are situated in the bottom right corner of the resulting pair. Because the unstable eigenvalues are uncontrollable, the corresponding rows in the transformed input matrix should be zero. Thus, after applying the transformations to the input and output matrices of $G_{i}^{-1} G$, the $n$th order outer factor results by simply retaining the subsystem corresponding to the first $n$ stable eigenvalues.

\section{Conclusions}

A completely general implementable procedure to compute J-inner-outer factorizations of rational matrices has been proposed. All computational steps of this procedure can be performed by using exclusively numerically reliable algorithms. The procedure is well suited for robust and modular software implementation. With $J=I$, it can be used to compute inner-outer factorizations too. It is worth to mention that the proposed procedure is applicable even when the given rational matrix has zeros on the imaginary axis for a continuoustime system or on the unit circle for a discrete-time system.

\section{References}

[1] B. A. Francis. A Course in $H^{\infty}$ Theory. Springer-Verlag, New York, 1991.

[2] H. Kimura. Conjugation, interpolation and model-matching in $H^{\infty}$. Int. J. Control, 49:269307, 1989.

[3] T. Katayama, K. Minamino, and N. Morihira. On the $J$-spectral factorization using descriptor transfer matrices. In Proc. 32nd CDC, pages 33043305, 1993.

[4] M. C. Tsai, C. S. Tsai, and Y. Y. Sun. On discrete-time $H^{\infty}$ control: A j-lossless coprime factorization approach. IEEE Trans. Autom. Control, AC-38:1143-1147, 1993.

[5] M. C. Tsai and L. W. Chen. A numerical algorithm for inner-outer factorizations for realrational matrices. Systems \& Control Lett., 20:209217, 1993.

[6] A. Ben Israel and T. N. E. Greville. Some topics in generalized inverses of matrices. In M. Z. Nashed, editor, Generalized Inverses and Applications, pp. 125-147. Academic Press, New York, 1976.

[7] T. Kailath. Linear Systems. Prentice Hall, Englewood Cliffs, N.J., 1980.

[8] A. Varga and P. Van Dooren. On computing generalized inverses of rational matrices with applications. In Proc. 1995 ICIAM, Hamburg, 1995. [9] P. Misra, P. Van Dooren, and A. Varga. Computation of structural invariants of generalized state-space systems. Automatica, 30:1921-1936, 1994.

[10] A. Varga. Computation of Kronecker-like forms of a system pencil: Applications, algorithms and software. TR R181-95, DLR-Oberpfaffenhofen, Inst. Robotics and System Dynamics, Jan. 1995.

[11] A. Varga. On stabilization of descriptor systems. Systems \& Control Letters, 24:133-138, 1995.

[12] A. Varga. Generalized Schur methods to compute coprime factorizations of rational matrices. In Proc. 1st Asian Control Conference, Tokyo, Japan, vol. 3, pp. 89-92, 1994.

[13] F. Y. Wang and M. J. Balas. Doubly coprime fractional representations of generalized dynamical systems. IEEE Trans. Autom. Control, AC-34:733$744,1989$. 